\title{
Left ventricular mechanical dyssynchrony graduation of myocardial perfusion gated SPECT phase analysis: What next
}

\author{
Nili Zafrir, MD, FESC, FASNC ${ }^{a}$ \\ a Cardiology Department, Beilinson Hospital, Tel Aviv University, Tel Aviv, Israel
}

Received Oct 8, 2016; accepted Oct 10, 2016

doi: 10.1007/s12350-016-0717-7

\section{See related article, pp. 999-1008}

The use of phase analysis from gated SPECT myocardial perfusion imaging (MPI) for the evaluation of left ventricular mechanical dyssynchrony (LVMD) was introduced by Chen et al. ${ }^{1}$

\section{BRIEF DESCRIPTION OF LVMD BY PHASE ANALYSIS}

Phase analysis was performed with Emory Cardiac Toolbox software (SyncTool $^{\mathrm{TM}}$, Emory University/Syntermed, Atlanta, Georgia, and USA). ${ }^{1}$ LV dyssynchrony was assessed by phase analysis. Fourier harmonic functions were used to approximate regional wall thickness changes over the cardiac cycle and to calculate the regional onset-of-mechanical contraction phase. After the onset-of-mechanical contraction phases were obtained 3-dimensionally over the left ventricle, a phase distribution map was formulated to represent the degree of LV dyssynchrony.

Normally, the left ventricle contracts in a coordinated manner so that most of the myocardial segments have nearly the same phase. Thus, the normal phase image is close to a uniform distribution, and the normal phase histogram is narrow and highly peaked. The quantitative indices of LV dyssynchrony were defined by five parameters, as follows: peak phase, phase standard deviation-SD, histogram bandwidth-BW

Reprint requests: Nili Zafrir, MD, FESC, FASNC, Cardiology Department, Beilinson Hospital, Tel Aviv University, Tel Aviv, Israel; zafrirmd@isdn.net.il

J Nucl Cardiol 2018;25:1009-11.

$1071-3581 / \$ 34.00$

Copyright (c) 2016 American Society of Nuclear Cardiology. (range), histogram skewness - S (asymmetry), and histogram kurtosis-K (peakedness). The normal limits of the 5 parameters were generated from a gated SPECT MPI study of 90 healthy subjects, 45 males and 45 females. ${ }^{1}$ These normal mean values were the basis for the ongoing studies.

Since then, many studies have been reported in the literature to assess the degree and severity of LVMD by phase analysis in different clinical subsets ${ }^{2-6}$ and with a specific attention to patients with LV dysfunction in order to select the optimal candidate for CRT. ${ }^{7-9}$

In the majority of the studies, one or two parameters of phase analysis from the five defined by Chen et al were used, which are as follows: histogram BW and/or SD, by mean values, were used for representing LVMD. ${ }^{10-12}$ These parameters, SD and BW, were found to be sensitive in selecting patients with heart failure and $\mathrm{LVEF} \leq 35 \%$ for $\mathrm{CRT}^{7-9}$

However, there is a wide range of variability in phase parameters (SD and $\mathrm{BW}$ ) in a so-called normal control population. This is because phase histogram is influenced by many factors, such as demographic (male, female), cardiovascular risk factors including hypertension, diabetes, renal disease, coronary artery disease. $^{10-13}$ These factors cause some difficulties in determining the exact normal values (mean or cut-off) of $\mathrm{SD}$ and/or BW for the individual. Reviewing the literature with the mean values of control group, which were defined slightly different from one study to another, for each of the parameters, it shows: the range of SD $\left(^{\circ}\right)$ values is between 7.6 and 12.2 to and of BW $\left(^{\circ}\right)$ values are between 26.1 and 36.5 . This variability may also be related to the imaging protocol and software. $^{14}$

Moreover, systolic LV function measured by LVEF is linearly correlated with LVMD as shown in many studies. ${ }^{12,13}$ The lower the LVEF, the higher the level of SD and BW, but there are exceptions. As such, in order to define the cut-off level of LVMD (differentiate 
between normal and abnormal), or normal mean level, we need to assess normal values of phase histogram, which take all the above variables into consideration. In comparison to small variation of SD and $\mathrm{BW}$ parameters seen in normal control studies, the mean values or $\mathrm{CO}$ values of $\mathrm{SD}$ and $\mathrm{BW}$ in patients with criteria for CRT are significantly higher: $\mathrm{SD}>40$ and $\mathrm{BW}>130$ (more than 4-6 times of normal), as shown in most of the studies.

In addition to the first normal control of Chen et al, who characterized the 5 phase parameters, only 2 groups studies examined the 4-5 parameters of phase analysis that represent LVMD. ${ }^{1,15-17}$ Trimble et al observed that $\mathrm{SD}, \mathrm{BW}, \mathrm{S}$, and $\mathrm{K}$ were significantly different between patients with LV dysfunction, LBBB, RBBB, and normal control group. ${ }^{15}$

Recently, two articles on the use of the four parameters of LVMD were published by RomeroFarina ${ }^{16}$ and the mechanical dyssynchrony gradation of these parameters by Aguade-Bruix. ${ }^{17}$ They studied the cut-off (CO) values of normal control versus patients with conduction defects $(\mathrm{CD})$, patients with mechanical cardiac disease, and patients with and without criteria for CRT. In a pilot study, they found that $\mathrm{CO}$ values of $\mathrm{SD}>18.4$ and $\mathrm{CO}$ of $\mathrm{BW}>51$ were the most sensitive parameters ( $75.5 \%$ and $78.7 \%$, respectively), and $\mathrm{CO}$ of $\mathrm{S} \leq 3.2$ and $\mathrm{CO}$ of $\mathrm{K} \leq 9.3$ were the most specific parameters (92\% and $94.7 \%$, respectively) between normal control group and the abnormal group including patients with CCD, MCD, CCD + MCD obtained by gated SPECT analysis including the four parameters. However, comparison between patients with and without criteria of CRT, the $\mathrm{CO}$ values were increasingly abnormal; $\mathrm{SD}>40.2, \mathrm{BW}>132, \mathrm{~S} \leq 2.3$, and $\mathrm{K} \leq$ 4.6. Of note, in these studies, the cut-off for each of the parameters of LV dyssynchrony was assessed by ROC analysis (and not by mean values) which assumed to be more accurate in detection cut-off point. It was done in normal population (all, women and men separately) and in each abnormal subgroup. They also found a significant linear trend between the degree of dyssynchrony of each parameter SD, BW, K, S with LVEF, and QRS duration.

In a continuation article of this group ${ }^{17}$ based on the same study population, the authors proposed a new concept based on a graduation of dyssynchrony which includes all the four parameters of phase analysis. As such, in Grade 1, only one of the four parameters is abnormal cut-off level, while in grade 4 , all the four parameters are abnormal (SD and BW above and S and $\mathrm{K}$ below the cut-off level). The authors suggested that the increased number of abnormal phase parameters ${ }^{1-4}$ is an indicator of increased dyssynchrony. For example, patients with group 4 graduations, all four parameters
(SD, BW, S, and $\mathrm{K}$ ) are abnormal. According to the authors, the $\mathrm{CO}$ graduation was found to be useful in selection patients for CRT. The discrimination between patients with criteria for CRT and without was strong.

The authors found that $92 \%$ of patients with criteria for CRT had 3 or 4 abnormal parameters in the phase analysis. This result may raise some question as if the rate of graduation is so high in almost all the patients with the accepted criteria for CRT, why should we need phase analysis. Probably, mean value or $\mathrm{CO}$ values for LVMD as well as parameter graduation should be considered in order to achieve high accuracy rate to predict benefit from CRT.

The authors concluded that $\mathrm{CO}$ graduation may assist in selection of patients for CRT on top of the accepted criteria. Of course, these results need validation with follow-up and outcome studies in patients after CRT installation.

In summary, since 2005, a large number of studies on phase analysis by gated SPECT MPI have been performed to assess LVMD in normal and in a variety of clinical subsets as well as in patients with LV dysfunction. It was found that $\mathrm{SD}$ and $\mathrm{BW}$ to be robust indicators of LVMD may serve as a predictive factor of both cardiac outcome and all-cause mortality among other predictors as NYHA classification, LVEF, scar size, and ischemia. ${ }^{10-14}$

Yet, the main reason to assess LVMD remains the need for better selection of patients who are appropriate candidates for CRT with high sensitivity and specificity. There is one prospective study by Henneman et al in 2007 , in a small group of 42 patients with severe LV dysfunctions who underwent $\mathrm{CRT}^{8}$ and were followed for response to CRT. They found an optimal cut-off value of 42 for SD (sensitivity and specificity of 74\%) and 135 for BW (sensitivity and specificity of 70\%) for the prediction response to CRT. However, this purpose has not been fulfilled yet. It seems that we have enough incentive to accomplish this task. What we need are the following:

1. A prospective multicenter study on patients with heart failure who were referred for CRT/ CRTD according to guidelines criteria, using gated SPECT including perfusion and function data and phase analysis - LVMD, of each of the four phase parameters and phase graduation in addition to echocardiographic assessment and QRS duration.

2. Follow-up studies of these patients after CRT, to assess cardiac outcome with relation to LVMD parameters, as well as improvement of heart failure, decreased life-threatening arrhythmias, and reduction of cardiac death,- -in other words, to increase the accuracy for detection responders to CRT. 
3. Finding out whether there is a yield for LVMD uses by phase analysis among the other criteria in patients prior CRT/CRTD, and what are the appropriate parameters of phase analysis to predict CRT benefit.

\section{Disclosure} interest.

The author declares that he has no financial conflicts of

\section{References}

1. Chen J, Garcia EV, Folks RD, Cooke CD, Faber TL, Tauxe EL, et al. Onset of left ventricular mechanical contraction as determined by phase analysis of ECG-gated myocardial perfusion SPECT imaging: development of a diagnostic tool for assessment of cardiac mechanical dyssynchrony. J Nucl Cardiol. 2005; 12:687-95.

2. Trimble MA, Borges-Neto S, Honeycutt EF, Shaw LK, Pagnanelli $\mathrm{R}$, Chen $\mathrm{J}$, et al. Evaluation of mechanical dyssynchrony and myocardial perfusion using phase analysis of gated SPECT imaging in patients with left ventricular dysfunction. J Nucl Cardiol. 2008;15:663-70.

3. Aljaroudi W, Aggarwal H, Venkataraman R, Heo J, Iskandrian AE, Hage FG. Impact of left ventricular dyssynchrony by phase analysis on cardiovascular outcomes in patients with end-stage renal disease. J Nucl Cardiol. 2010;17:1058-64.

4. Atchley AE, Trimble MA, Samad Z, Shaw LK, Panaganelli R, Chen J, et al. Use of phase analysis of gated SPECT perfusion imaging to quantify dyssynchrony in patients with mild to moderate left ventricular dysfunction. J Nucl Cardiol. 2009;16:888-94.

5. Aljaroudi WA, Hage FG, Hermann D, Doppalapudi H, Venkatarama R, Heo J, Iskandrian AE, et al. Relation of left ventricular dyssynchrony by phase analysis of gated SPECT images and cardiovascular events in patients with implantable cardiac defibrillators. J Nucl Cardiol. 2010;17:298-304.

6. Goldberg AS, Alraies MC, Cerqueira MD, Jaber WA, Aljaroudi WA. Prognostic value of left ventricular mechanical dyssynchrony by phase analysis in patients with non-ischemic cardiomyopathy with ejection fraction $35-50 \%$ and QRS $<150$ ms. J Nucl Cardiol. 2014;21(1):57-66.

7. Boogers MM, Van Kriekinge SD, Hennemam MM, Ypenburg C, Van Bommel RJ, Boersma E, et al. Quantitative gates SPECT derived phase analysis on gated myocardial perfusion SPECT detects left ventricular dyssynchrony and predicts response to cardiac resynchronization therapy. J Nucl Med. 2009;50:718-25.
8. Henneman MM, Chen J, Dibbets-Schneider P, Dibbets-Schneider P, Stokkel MP, Bleeker GB, et al. Can LV dyssychrony as assessed with phase analysis on gated myocardial perfusion SPECT predict response to CRT? J Nucl Med. 2007;48:1104-11.

9. Henneman MM, Chen J, Ypenburg C, Dibbets P, Bleeker GB, Boersma E, et al. Phase analysis of gated myocardial perfusion SPECT compared to tissue Doppler imaging for the assessment of left ventricular dyssynchrony. J Am Coll Cardiol. 2007;49:170814.

10. Uebleis C, Hellweger S, Laubender RP, Becker A, Shon HY, Lehner S, et al. Left ventricular dyssynchrony assessed by gated SPECT phase analysis in an independent predictor of death in patients with advanced coronary artery disease and reduced left ventricular function not undergoing cardiac resynchronization therapy. Eur J Nucl Med Mol Imaging. 2012;39:1561-9.

11. Pazhenkottil AP, Buechel RR, Husmann L, Nkoulou RN, Wolfrum M, Ghadri JR, et al. Long-term prognostic value of left ventricular dyssynchrony assessment by phase analysis from myocardial perfusion imaging. Heart. 2011;97:33-7.

12. Zafrir N, Nevzorov R, Bental T, Strasberg B, Gutstein A, Mats I, et al. Prognostic value of left ventricular dyssynchrony by myocardial perfusion gated SPECT in patients with normal and abnormal left ventricular function. J Nucl Cardiol. 2014;21:53240.

13. Hess P, Shaw L, Fudim M, Iskandrian A, Borges-Neto S. The prognostic value of mechanical left ventricular dyssynchrony defined by phase analysis from gated single photon emission computed tomography myocardial perfusion imaging among patients with coronary heart disease. J Nucl Cardiol. doi: 10.1007/s12350-016-0423-5.

14. Aljaroudi W, Jaber WA, Cerquera MD. Effect of tracer dose on left ventricular mechanical dyssynchrony indices by phase analysis of gated single photon emission computed tomography myocardial perfusion imaging. J Nucl Cardiol. 2012;19:63-72.

15. Trimble MA, Borges-Neto S, Smallheiser S, Chen J, Honeycutt EF, Shaw LK, et al. Evaluation of left ventricular mechanical dyssynchrony as determined by phase analysis of ECG-gated SPECT myocardial perfusion imaging in patients with left ventricular dysfunction and conduction disturbances. J Nucl Cardiol. 2007;14:298-307.

16. Romero-Farina G, Aguade-Bruix S, Candell-Riera J, Pizzi MN, Garcia-Dorado D. Cut-off values of myocardial perfusion gatedSPECT phase analysis parameters of normal subjects, and conduction and mechanical cardiac diseases. J Nucl Cardiol. 2015;22:1247-58.

17. Aguade-Bruix S, Romero-Farina G, Candell-Riera J, Pizzi MN, Garcia-Dorado D. Mechanical dyssynchrony graduation according to validated cut-off values of myocardial perfusion gated SPECT phase analysis parameters. JNC-16-198-OA.R2 